\title{
GEOSPATIAL DATABASE UPDATING SYSTEM with WMS and DIRECT CONNECTION METHOD
}

\author{
B. Yüksel ${ }^{1}$, A. Yilmaz ${ }^{1, *}$ \\ ${ }^{1}$ General Command of Mapping, Photogrammetry Department, 06590 Dikimevi Ankara, Turkey - (bekir.yuksel, \\ altan.yilmaz)@hgk.msb.gov.tr
}

Commission IV, ICWG IV/III

KEY WORDS: TOPOVT, Updating of Geodatabase, Topographic Database, WMS, Direct Connection

\begin{abstract}
:
The demand for up-to-date geospatial data is in an upward trend. This brings a continuous and sustainable review and update of the geospatial data itself and database design. Turkish Topographic Vector Database (TOPOVT) is the main database of Turkey consisting 1:25.000 and higher scale resolution 3D topographic vector data covering whole country. It also consists of contours and geonames. TOPOVT has been and is still being produced by General Command of Mapping of Turkey mainly from $30 \mathrm{~cm}$ resolution stereo aerial photos and completed in the field. TOPOVT is seamless and topologic database. The updating objective is five years cycle. The first production cycle of TOPOVT in vector has been completed recently. Before the updating cycle began, a system was designed to update TOPOVT efficiently and without losing any information content. It also holds historical background of the features updated.
\end{abstract}

With this topographic data update system, geospatial data can be updated rapidly and served to the users. Hereby both the process for map printing fastens and answers to the need of updated data for the TOPOVT database can be achieved. With this system, the data which will be updated can be displayed on the personal computer by TOPOVT database connection and the users can perform add, update and delete actions in the data according to their authorization. All the updates executed in the field can be monitored on the TOPOVT database in real time via internet connection.

\section{INTRODUCTION}

Earth's surface is changing continuously due to natural and/or artificial factors. While natural change is slow, artificial/man made change occurs more rapidly. Especially in developing countries these changes take place even faster. In order to keep up pace with the changing situations, decision makers have to be informed on time geospatially (Maras and Altan, 2000). The geospatial data can be updated by field survey, remote sensing techniques, compilation, or by comparing existing data by a new reference (Samen et al., 2014).

Classical methods cannot keep up pace with the speed of geospatial data change. Some researchers are put forth methods for geospatial data updating. Pan et al., (2014) discussed raster to raster data, raster to vector data and vector to vector data update technology. They claimed that these methods are efficient and accurate enough. Lopez-Pellicer et al., (2011) looked for the web based geoprocessing and Open Geospatial Consortium (OGC) means of updating geodata. They found that Web Processing Service (WPS) compliant features are lacking in data providers and service providers have to provide semantic interoperability in geospatial systems. Samen et al., (2014) proposed a new method in order to enter geospatial data to database by HTML Object Library and Open Source GIS. They transferred the data to geospatial database by an interface.

Maras and Altan (2000) proposed the history of the update process has to be stored. They inserted that deleted features had to be accessible for their information content. They also suggested that feature addition and updating should be included in their attributes.
Pla and Lleopart (2010) asserted the collaborative updating by using OGC, Geographic Markup Language (GML) and Web Feature Service (WFS) standards. They tried to coproduce The Institut Cartogràfic de Catalunya (ICC) vector data with shareholders. They also improved ICC geospatial databases by means of the data model improvement holding unique identifiers and attributes for versioning and step-by-step updating. They hoped for quality enhancement and wide usage of geospatial databases.

Turkish Topographic Vector Database (TOPOVT) is the geospatial database consisting of topographic features in 1:25.000 and larger scales covering whole Turkey. So far, TOPOVT is produced by General Command of Mapping. Y1lmaz and Caniberk (2018) proposed a web based updating system for TOPOVT. This real time updating system allows shareholders to facilitate and update TOPOVT by an internet browser with a direct connection to the geospatial database.

When a vector database link is established on Esri ArcGIS, all the geodata is on the screen and the database is busy with this query as in other Geographic Information Systems (GIS) software. If the size of the mentioned data is large, it will cause slowdown both in display and update operations. Since this slowness prevents updating the data quickly and effectively, a more efficient method of updating TOPOVT, which includes continuous large size data covering the whole country have been searched for.

As a result of the analyses made; it is determined that there is no need to be present on the screen as a vector if the update is not performed first. If the user wants to update the data that he / she has reviewed, it is thought that it can be an effective method to 
update the data by calling the prepared program and send it back to the database at the end of the process.

In order to implement this method, it is necessary to transform the current version of the data into its own symbols and to use it as a vector to be updated, if necessary. The design of the system was carried out in three main groups related to each other. These are:

1. Preparing the WMS service which will bring the appropriate images of the data to the screen at the desired scales,

2. Designing the personal vector database in the user side and making the necessary changes and additions in the enterprise vector database,

3. Development of the software that will provide the link between the WMS publication and the vector data structure prepared in the first and second articles.

\section{PREPARATION OF WMS SERVICE}

All existing vector data in the database is added to ArcMap screen and WMS service is prepared by using its own symbols. In order to provide vector data link over this service later, some rules were put in the layer naming. These rules are as follows:

1. The layer names will be identical to the name of the corresponding feature class. For example, the layer name that receives data from the "FOREST" feature class must begin with the FOREST statement.

2. The "-" sign will be used to distinguish the expression to be used after the feature class name.

3. After the feature class names: if it is desired to write active and passive scale ranges on the screen then the values will be written as 3 missing and with a "_" sign in between. 4. The expressions used with the "_" sign tell what the features of the feature class are.

According to these rules, an example layer naming scheme is: The name "MUNHANI-15_5_ANA" is the name of the "CONTOUR" layer and the feature class that the layer receives data from. The "-" sign indicates that the subsequent statements are meant to be informative. "15" refers to a scale of 1: 15,000, and " $5 "$ refers to a scale of 1: 5,000.

These values provide information about the minimum and maximum scale ranges that the layer will occupy on the screen. The interval at which the layer is shown is already present in the properties of the WMS layer. The purpose of writing these values is to ensure that the layers that are not included in any group layer can be directly distinguished by the names of the minimum and maximum scale ranges. "ANA (means MAIN)" is the name of the subtypes of the features shown on this layer. This demonstration is intended only to prevent the mess that will occur when there are only 2 layers in the same name. Which features are shown are already present in the "Description" section of the layer's properties as an SQL statement. For example, the "Description" value of this layer is "topodetayalttipno $=4 "$. (Figure 1) How this phrase is used will be explained in detail in the next section.

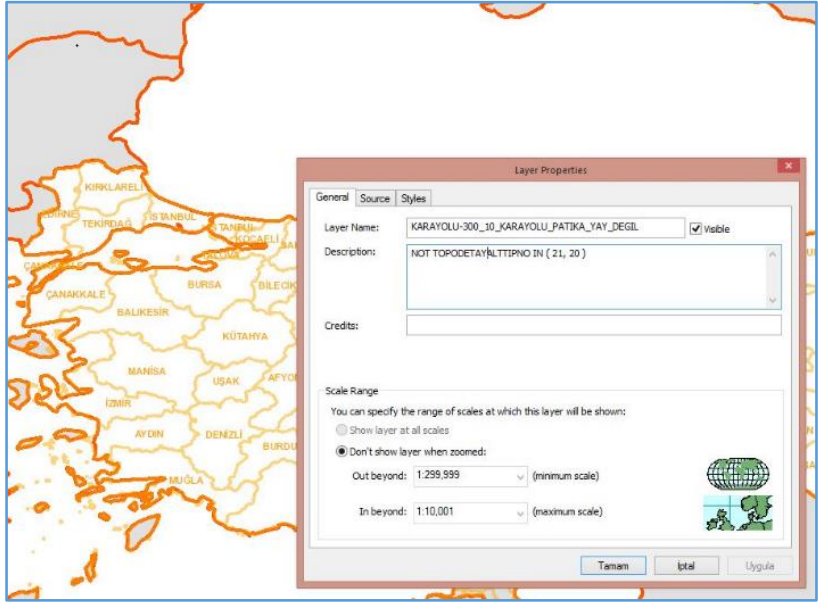

Figure 1. Layers and properties of WMS service

The corporate database version from which the data is retrieved is determined to be an upper version of the version that the user will be working on. The purpose of this is to ensure that the service changes after the system administrator has performed the checks. For example, when the WMS service version is "UPGRADE", the user workgroup is "Topog" and the version that the operations are done and recorded is a lower version opened with the user's name on the site. (Figure 2) Features at the end of each process: if the database is available, it is sent to the next version and if any changes made by other users are received. (Reconcile and Post operations) The changes sent by the users to the "Topog" group are changed to the

"GUNCELLEME" version (Reconcile and Post operations) after being controlled by the system administrator, or the WMS publication is also changed directly to the main version. The versions that users query for and change the features remain their same version until the mentioned submission is realized.

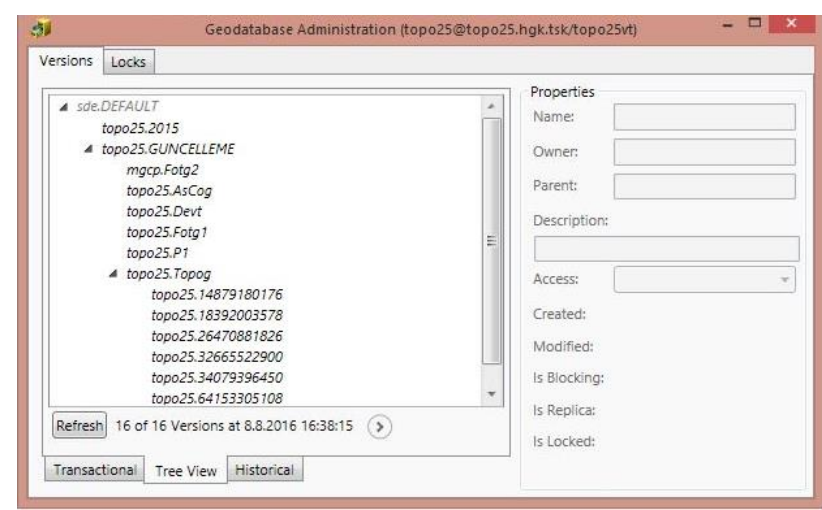

Figure 2. WMS service version hierarchy

The symbols shown on the printed map are used in the display of the features. The minimum and maximum scales for each feature class have been determined and published as WMS via ArcGIS Server so that the user can display all feature classes effectively (Figure 3). 


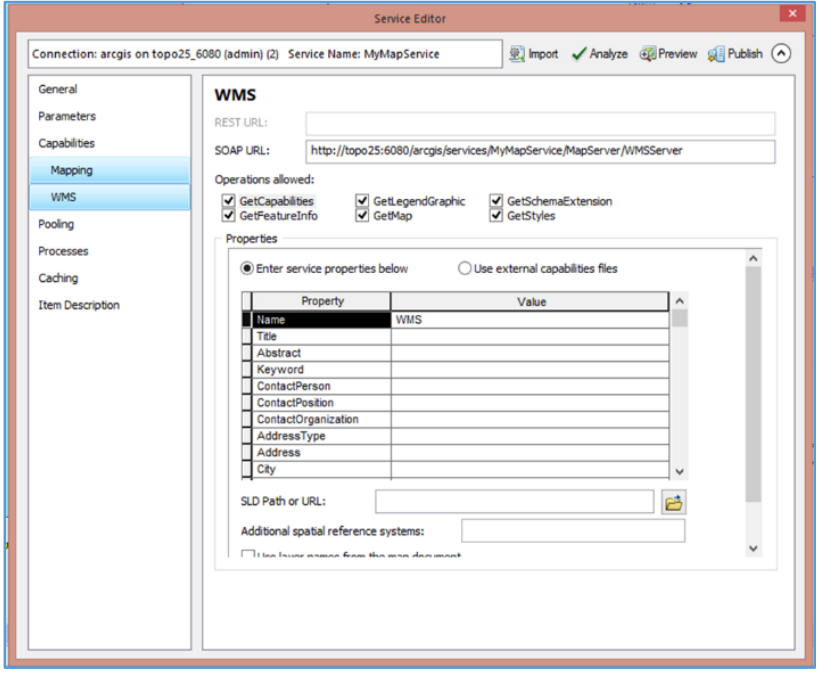

Figure 3. WMS service

All TOPOVT database feature classes are included in the WMS service. As it is known, the data in these feature classes are used for 1: 25.000 scale map production. Therefore, in order to be able to show the features in readable condition at every scale, model generalization was made by determining appropriate scales for each feature class display. An example of the generalization of the constructed model is as follows: "GENISDERE-

2200_250 "layer shows features from the" GENISDERE "feature class on the screen with" st_length (shape)> 2 "description value. The minimum display scale is $1: 2,200,000$ and the maximum display scale is $1: 250,000$. This feature class only takes features with a length greater than 2 degrees. The demonstration of features with a length of less than 2 was meaningless because it would be very difficult to detect in this scale range.

This service is both reinforced and backed up by means of a system which is independent from one another but at regular intervals. If one of these two services becomes unavailable, the other will be able to meet the demands. In addition, when two systems are in use together, load-balance process is shared by load balancing software to load user requests on a single server (Load-Balance Process).

\section{CHANGES IN VECTOR DATA STRUCTURE}

Changes in vector data structure were performed in two groups. These are:

1. Preparation of personal database with temporary workspace,

2. Changes to the Enterprise Database.

\subsection{Preparation of Personal Database with Temporary Workspace}

When the user calls vector data from the database, a schema copy of the database is created in the folder named TOPO25LOG in the first drive (D, E, F etc.) after the system driver in the ESRI File Geodatabase format opened with the user's own name. This personal database is designed as a temporary storage medium for vector data until the update process is complete (Sending Process). In this personal data base, most of the feature classes used in WMS service are used. The feature classes in these are the feature classes in the feature data set called "Topo25DetayVeriSeti" in the original corporate database. This is a small copy of the enterprise database schema with "ISLEMID (Process ID)", "DELETE" attribute fields and metadata fields ("OLUSTURAN (creator)", I "OLUDURDATA", "DEGISTIRMETARIHI (modification date)") added to every feature class in this file. The properties of the "ISLEMID" attribute field are the same as in Figure 4.

Field Properties
\begin{tabular}{|l|l|l|}
\hline Alias & ISLEMID & \\
\hline Allow NULL values & Yes & \\
\hline Default Value & & \\
\hline Length & 400 & \\
\hline
\end{tabular}

Figure 4. ISLEMID field properties

The ISLEMID attribute field is the recordable area for the software that will be described in the third item. The "GLOBALID" value in the corporate database is recorded in feature's "ISLEMID" attribute field called from the enterprise data base.

In the "SILINDI (deleted)" attribute field, information about whether the deleting process has been performed or not has been kept. Features are deleted from the database using the information in this area during the sending process. In addition, the metadata information of all changes made using the "ArcGIS Editor Tracking" feature is instantaneously retained. Metadata areas are as follows: "OLUSTURAN", "OLUSTURMATARIHI (creation date)", "DEGISTIREN (modifier)", "DEGISTIRMETARIHI (modification date)".

\subsection{Changes to the Enterprise Database}

In an enterprise database, the attribute field value of the feature class called "OBJECTID" is only a unique number for that database. If the database being run is replaced with another replica, the value "OBJECTID" loses its unique property in that feature class. Because when the institutional database is changed, the values of the feature class "OBJECTID" field will also be specific to that database. In order to avoid this confusion, a number is given when the institutional database is changed to "GLOBALID" for all feature classes. This process, which is already a requirement for the replication of the enterprise database, has been done automatically by ArcGIS software.

After a unique numbering of the features, a 4-step change has been made in order to ensure that the record of the changes to be made directly in the database is correct.

The first of these is to provide feature-based metadata fields to track data entries through the attributes of feature. These areas are shown in Table 1. The fields "KAYNAK (source)", "KAYNAKTARIHI (source date)", "GUNCELLEYEN (updater)" and "GUNCELLEMETARIHI (update date)" are the fields designed by the software to be submitted during the sending process. 


\begin{tabular}{|l|l|l|l|}
\hline $\begin{array}{l}\text { KAYNAK } \\
\text { (String) }\end{array}$ & $\begin{array}{l}\text { KAYNAKTA } \\
\text { RIHI } \\
\text { (Date) }\end{array}$ & $\begin{array}{l}\text { GUNCELLEYEN } \\
\text { (String) }\end{array}$ & $\begin{array}{l}\text { GUNCELLEMETARIHI } \\
\text { (Date) }\end{array}$ \\
\hline KYGM & $\begin{array}{l}15.12 .2015 \\
12: 11: 55\end{array}$ & 12345678912 & 20.12 .2015 13:11:34 \\
\hline Topog & $\begin{array}{l}12.12 .2015 \\
12: 11: 55\end{array}$ & 12345678912 & 19.12 .2015 13:11:34 \\
\hline
\end{tabular}

Table 1. Feature based metadata field examples

The second change has been to create feature classes that will hold the previous states of the features in each updating process, in order to ensure that the changes made to the institutional database can be interrogated temporally. This feature class naming was done using the feature class name and the "_DEGISIM (change)" annex. All of these classes are again included in a separate data set using the "_DEGISIM (change)" plugin. For example, for the "ORMAN (forest)" feature class changes in the "Topo25DetayVeriSeti" data set, the "ORMAN_DEGISIM (forest change)" feature class was created in the "Topo25DetayVeriSeti_DEGISIM" data set. These change classes are a blank copy of the original classes as a schema. It is possible to see the features of this feature on both geometric and featured dates at different times (Figure 5).

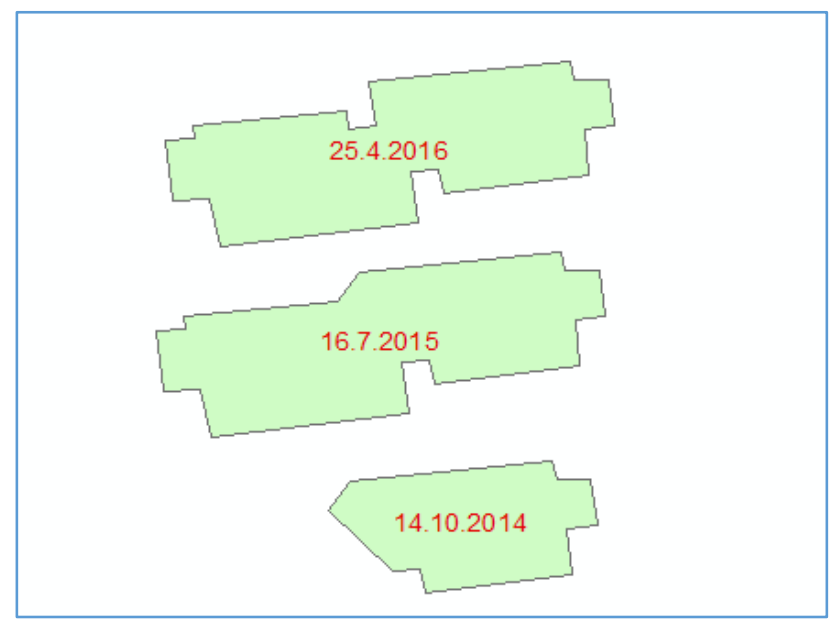

Figure 5. Temporal changes in "BUYUKBINA (building) feature class

The third change is to make an instant backup and create coservers to ensure that the system runs seamlessly in the enterprise database. In this way, two completely independent systems can be stored in corporate databases for both WMS broadcast and vector data update.

Also, the constants used by the software that will be mentioned in the following sections are also recorded in the "PARAMETERS" table available in the database. Thus, it will be sufficient to change the constants in this table without having to resort to writing for a change to be made at a later time.

\section{DEVELOPMENT OF SOFTWARE BETWEEN WMS SERVICE AND VECTOR DATA STRUCTURE TO PROVIDE CONNECTION}

Users are asked to $\log$ in to the system with their usernames before the update process starts (Figure 6). Once logged in, the user chooses the database username, the coordinate system to work, the database version and the server to work in the given authorities. The enterprise database connection created with these selections is the connection to query, invoke and send vector data. The value of "TC Kim.Nu (national ID)" in this area constitutes the value of the field named "GUNCELLEYEN" in feature-based metadata and "VT Vers." value of the field named "KAYNAK" (Table 1 and Figure 6).

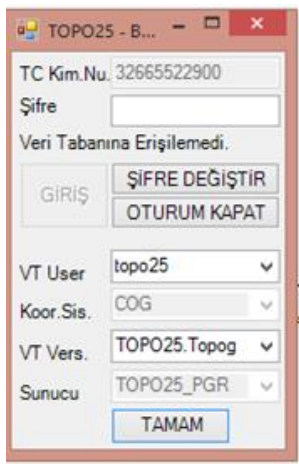

Figure 6. User entry

The connection between the WMS publication on the ArcMAP screen and the corporate database vector data is also made through two plug-ins running on ArcMAP software. These extensions are the keys labeled "CAGIR (call)", which are transferred to the personal database of File GeoDatabase in the computer, and "GONDER (send)" keys, which are used to send the data back to the corporate database after the update is completed.

The functions of these keys will be explained in 4 steps in the workflow during the updating process. These stages are:

1. Calling the WMS service and examining the features,

2. Calling some of the features examined,

3. Modification of the called feature or the addition of new features,

4. Posting the completed changes to the relevant feature classes.

\subsection{Calling the WMS service and examining the features}

The "CAGIR" key is pressed on the ArcMAP interface for the purpose of calling the corresponding broadcast. If there is no broadcast on the screen that matches the WMS address registered in the "PARAMETRELER" table in the corporate database, it is added. This process is repeated by pressing each "CAGIR" key.

The data call key also makes it possible to make the "ISLEMID" field of this class table invisible to the user if one of the feature classes in the personal database mentioned in the previous sections on the screen is on the screen. Thus, the changes made by mistakenly can be prevented.

\subsection{Calling some of the features examined}

If there are features or features to be called, the "CAGIR" key is pressed first. When this key is pressed, the mouse pointer changes to match the feature call. It can be selected by clicking on a single point or by region. When a single click is made with the mouse, a 5-meter-radius query area centred on the click-point is created and the attributes of the first feature entering the query area are 
displayed (Figure 7). In this questionnaire, which feature classes are determined according to the geometry type of the feature class to be interrogated. Point, line, annotation and field features are checked in order. This class of feature is taken to the beginning of the geometric type feature class list in order to gain speed in subsequent queries, if any data in the feature class is found. For example, the "TARLABAHCE (field and garden)" feature class shown in Figure 7 has been taken into account within the field feature classes group. As a result of repeating these operations during the query, the most frequently used feature classes will be listed at the top of the lists and the results will start to get faster.

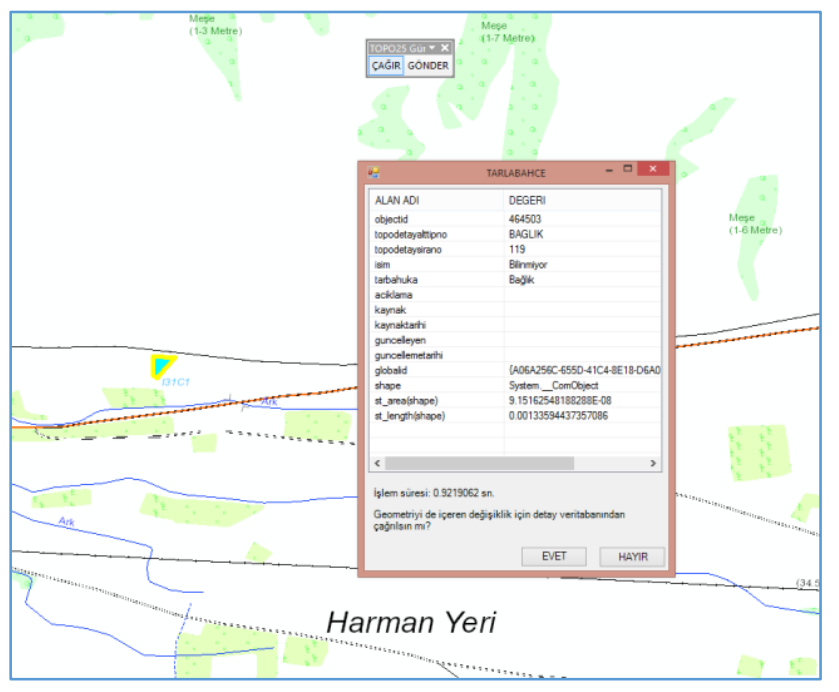

Figure 7. Displaying single feature's attributes

If a regional selection is desired, a query box is created by dragging the mouse. This query box becomes a box with a yellow stripe on the screen, and a red line box when it reaches a certain size. Turning to rich red means that regional selection can now be made (Figure 8 ). The value that determines this change is the value in pixels square of the selection field (width * length). If this value is greater than the value in the "PARAMETRELER" table, it turns red and the regional selection becomes ready. The only difference from the single feature selection of the yellow boxed state is that it determines the query area more precisely.

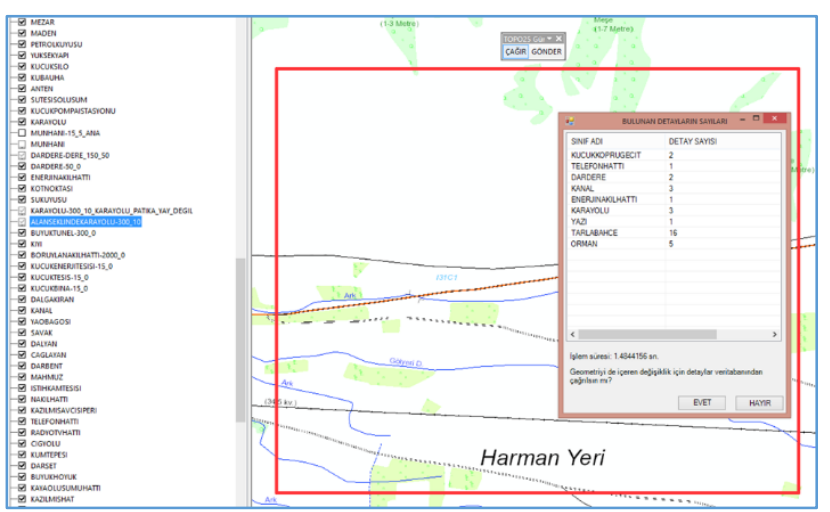

Figure 8. Number of features in selected area

The query in the selection field in Figure 8 is made on all layers selected in the layer list of the WMS publication. The current screen size does not take into account whether the layer is active or not. For example, since the "MUNHANI (contour)" and
"MUNHANI-15_5_ANA" layers are not selected in Figure 8, the layers "DARDERE-DERE_150_50 (narrow stream)", "KARAYOLU-300_10_KARAYOLU_PATIKA_YAY_DEGIL (highway)", "ALANSEKLINDEKARAYOLU-300_10 (area highway)" are quarried because they are selected even though they are not passive. The number of features in which feature class is shown on the screen.

If the features of the feature attributes or multiple selection are displayed in the form shown in Fig. 7 and Fig. 8 where the feature numbers are displayed, the "EVET (yes)" key is clicked to call the features. The data as given in section 3.1 is temporarily copied to the user's computer. With this copy, the data output for each feature will be saved in the "3.2. Changes Made in the Enterprise Database "section, as described in the "GUNCELLEME (update)"table. The classes of the feature invoked after copying the features are added to ArcMAP as a layer.

As described in section 4, the enterprise database connection to be run when the user enters the system is determined. The version specified on this connection is the name of the main group that the name user will use during the invocation process. When the call key is first pressed, it is checked whether this version of the main group is a version opened under the user's name, otherwise it is created. This version makes every time the "CAGIR (call)" key is pressed and it is equalized (RECONCILE POST operation) if the connection version which is an upper version is available. Since each sync will also be synchronized, it means that changes sent from other versions of this same group are also transferred to that user's version. That is, if the quarried or called feature is sent to the database, it includes the changes of other users.

\subsection{Modification of the called feature or the addition of new features}

The changes on the invoked features are grouped under three headings: attribute feature or geometric update (UPDATE), deletion of existing feature (DELETE) and creation of new feature (INSERT). An attribute or geometric update of an existing feature is a change to be made on the called feature. At the same time, this feature may become fragmented and represented in more detail. Features can be deleted before or after shredding. When new feature is added, if it is desired to add feature to the feature class, that feature class is opened and new feature is created.

Because the "KAYNAKTARIHI (source date) attribute is the ArcGIS Editor Tracking field, it is automatically filled in by ArcGIS when it is modified by the user and cannot be changed by the user. All these operations take place on the user's computer after the features are called from the corporate database.

\subsection{Posting the completed changes to the relevant feature classes}

If the changes made after the completion of user changes are to be sent to the enterprise database, the feature must be attached to the ArcMAP screen first. The "GLOBALID" value in the feature "ISLEMID" attribute field contains the relevant feature to be updated in the corporate database.

We can collect the situations to be encountered in sending out in five groups. These are: 
1. If the feature record is not in the database,

2. If there is no change in the feature,

3. If geometric or attribute change is detected in the feature,

4. If the called feature is deleted,

5. If a new feature is created.

If the features are not in the enterprise database, there are three possibilities. The first is that the feature output that the user is invoked has been cancelled by the system administrator. Second, the corporate database has changed and the changes in the other enterprise database have not been transferred to the database used in the related versions. This occurs when the enterprise databases used are changed for maintenance activities. In the third case, the values in this area may be corrupted by the user. In these three cases, the system is directed to the administrator in the transaction report and the feature is deleted from the personal database. No further processing is required if the feature recording is intentionally deleted by the system administrator. As mentioned above, if a corporate database change is mentioned, reconcile-post operations and replication operations are performed by the system administrator and the related record is cleared. This is not the case if these operations were done before the corporate database change and all changes were made to the corresponding version.

If there is no change in feature, the feature is deleted from the temporary personal database.

If geometric or attribute change in feature is detected; the software determines whether the changes are geometric or attribute. This information is used only for reporting the transactions made. The registration in the detailed corporate database is transferred to the "DEGISIM (change)" feature classes, and the feature of the submitted features is changed with the registration in the corporate database. If the called feature is divided into more than one features, each feature is recorded separately in the corporate database. The divided features are again determined according to the "ISLEMID" value.

If the invoked feature is deleted, its detection is done using the "SILINDI (deleted)" attribute field. The feature is transferred to the "DEGISIM (change)" feature class in the corporate database as it was before the features were changed.

Whether it is an existing feature or a new feature is decided by looking at the "ISLEMID" field. If there is no value in the "ISLEMID" field (Null) or if it consists only of a space character, this feature is detected as a newly added feature in the related feature class and saved as such.

\section{SUMMARY}

To summarize the operations described so far, briefly; two independent enterprise database systems were established and synchronization was achieved at short intervals. These systems, which are the backups of each other, have the same 2 WMS broadcasts, and these broadcasts are broadcast over a single address. Thus, if one of the systems fails or is taken out of the way, the updating process is not affected. In addition, since the features of the update are made in the temporary files on the personal computers, the operations on the features have been made extremely simple.

The metadata of the features are filled in by the system as described in chapter 3 . When the user logs in to the system, the "GUNCELLEYEN (updater)" and "KAYNAK (source)" attribute fields are filled (Figure 9). Detailed information about the version used can be found in the enterprise database in the table "VT_VERSION". For example, in the description of the KYGM version, the expression "consists of data received from the General Directorate of Highways on February 13, 2015". "KAYNAKTARIHI (source date)" is the last date the feature was changed as mentioned in section 3, and "GUNCELLEMETARIHI (update date)" is the date when the feature was returned to the system.

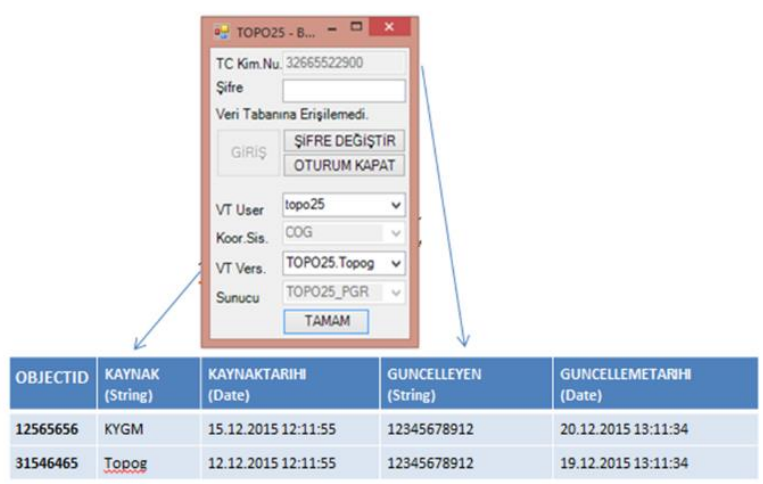

Figure 8. Feature based metadata

\section{CONCLUSION}

Although widely used GIS software has a great deal of vector editing capability, the ability to quickly and accurately update the database depends on the speed of direct connection or WFS services. This hybrid system was created using limited direct linking over WMS service as an effective data entry method that does not give up the comprehensive and effective vector data editing capabilities of GIS software.

Geospatial data update ability was gained by the proposed system to TOPOVT which is the geospatial database covering whole Turkey other than file-based production. An alternative way to update any desired layer or feature of any desired region on this page quickly and efficiently has been established. In addition to this system, which is developed by adding plugin to ArcGIS software which is currently being used, the display convenience of WMS service and the ability of editing WFS service vector data are combined. At the same time, when the data operator is able to see the vector data having a tight topological relation with each other in an integrated manner with their own symbols on the WMS service, features are prevented from reaching the vector data through the WFS service unnecessarily by slowing down the system. Thanks to the fact that the system does not directly access the vector database, it grows as the database grows, ensuring that the update system is not affected.

It is known that many institutions and organizations are trying to have methods to update their central databases quickly and effectively. Given the high cost of licensed GIS software, the vast majority of these systems aimed at reaching a large number of data producers are concentrated on web-based systems. If these websites are a significant amount of resources spent, they focus on geospatial data viewing, basic GIS capabilities and user authorization. Well, can an open-source software that already possesses a lot of these capabilities enable thousands of vendors to enter data? How well can these web systems, which seem adequate for basic operations, respond to the needs of specialized data producers? 
These questions are considered to be compatible with any open source code (QGIS, SAGA, GRASS ...) while this system is developed using ArcGIS libraries. Together with the use of open source GIS software is rapidly increasing in public institutions nowadays, this proposed system can be applied to collecting and updating vector data with other open source software in large geospatial data producer organizations.

\section{REFERENCES}

Lopez-Pellicer, F. J., Rentería-Agualimpia, W., Béjar, R., Valiño, J., Zarazaga-Soria, F. J., and Muro-Medrano, P. R. 2011. Implantation of OGC geoprocessing services for Geoscience. In Proceedings of the II Iberian Conference of Spatial Data Infrastructures (pp. 1-12).

Maras, H. H., and Altan, M. O. 2000. Updating of a Geographic Database: an Application and Design of a Geographic Information System. International Archives of Photogrammetry and Remote Sensing, 33(B4/2; PART 4), 616-623.

Pan, J., Xu, Q. and Yang, C. 2014. Research and Application of GIS Data Update Technology. The International Archives of Photogrammetry, Remote Sensing and Spatial Information Sciences, 40(4), 195.

Pla, M., and Lleopart, A. 2010. Updating of vector databases at the Institut Cartogràfic de Catalunya. Catalonia, 1(5), 000.

Sameen, M. I., Ali, A. A., Wahid, N. O. A., and Kubaisy, M. A. A. 2014. An Approach to Develop a Geographic Information Database Using Dot Spatial Open Source Platform and Google Search Engine. J Comput Sci Syst Biol, 7, 217-220.

Yilmaz, A., and Caniberk, M. Real Time Vector Database Updating System: A Case Study for Turkish Topographic Vector Database (TOPOVT). International Journal of Engineering and Geosciences, 3(2), 73-79. 